\title{
COMPARISON OF CONTRASTING METHOD BASED ON LOCAL CONTRAST MEASUREMENT
}

Some biological experiments and techniques, especially electron microscopy, require special sample treatments. These treatments usually change the samples and biologist cannot apply different techniques on the very same sample. In this case, it is necessary to have some comparison method to compare different treatments. In the electron microscopy, the heavy metals are used as contrasting chemicals. Question is which contrasting method is the best or which is better for imaging of given samples. Standard method is based on visual comparison and it is user dependent. We propose to measure local contrast and some other values like SNR (Signal-to-Noise Ratio), histograms, intensity profiles on the borders of the object and calculate statistical values for pixels' intensities.

Keywords: TEM images, local contrast measurement, image comparison, biological samples, SNR.

\section{Introduction}

Imaging of biological samples using the TEM (Transmission Electron Microscope) working at the accelerating voltage 100 $\mathrm{kV}$ requires usually an enhancement of contrast. This is achieved by addition of heavy metals, like $\mathrm{Os}, \mathrm{U}, \mathrm{Pb}$, etc., into the cell structure during the staining steps of the specimen preparation [1]. The next question is how to compare the resulting contrast of selected organelles in the TEM images.

The contrast or contrast resolution is defined as ability to distinguish between differences in intensity values of the object or area of interest and its surroundings in the image [2]. In our case, we are trying to find methods which will increase the difference between organelles and the rest of the cell and also between organelles themselves. The standard way of comparison is that user looks at the images and says what is better. This is of course very subjective and almost irreproducible measurement (observation). The question is how to measure local contrast in the image. There are several methods using the SNR (Signal-toNoise Ratio), RMS Contrast or others [3, 4]. But not all of them are useable for the TEM images due to the high level of noise. And many of them are only for evaluation of the whole image.

\section{Materials and methods}

To determine the difference between contrasting methods we need to define some ratio or some scale. Contrast of the object depends on the intensity of this object and on the intensity of its surroundings. We developed a tool, which provides us with the basic statistical analysis of these values. It is necessary to select the object of interest manually but the rest of the analysis is done automatically. The automatic detection does not work properly due to the high magnification, high level of noise and fuzzy borders of the object.

The decision about which method is better will not be made based on the SNR nor difference between the value of average intensity of the object and its neighbourhood, this can be misleading, but on the contrast value, calculated based on the Michelson contrast:

$C=\frac{L_{\max }-L_{\min }}{L_{\max }+L_{\min }}$

where $L_{\max }$ and $L_{\min }$ are the highest and the lowest luminance [5]. We cannot use the maximum and minimum luminance or intensities because of the high level of noise. We decided to change the Michelson formula to use the average (or median) values of intensities:

$C=\frac{I_{O}-I_{B}}{I_{O}+I_{B}}$

\footnotetext{
* Tomas Nahlik

The Institute of Technology and Business in Ceske Budejovice, Faculty of Technology,

Department of Informatics and Natural Sciences, Czech Republic

E-mail: nahlik@mail.vstecb.cz
} 


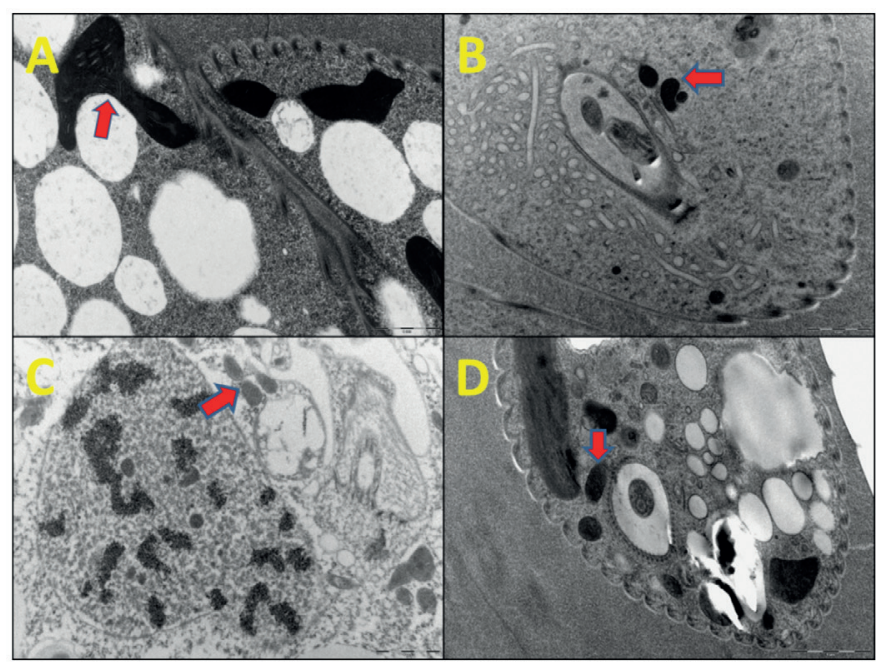

Fig. 1 Images of Euglena gracilis using different contrasting methods: $A$ - $2 \% \mathrm{OsO}_{4}$ in $100 \%$ acetone; $B$ - OTO (O-osmium, T-thiocarbohydrazide, O-osmium) method (2\% $\mathrm{OsO}_{4}, 100 \%$ acetone, thiocarbohydrazide); $\mathrm{C}$ - OTO plus $0.1 \% \mathrm{UA}, \mathrm{Pb}\left(\mathrm{NO}_{3}\right)_{2}$, and methanol;

$D$ - Ferrikyanide plus OTO and $0.1 \%$ UA

where $I_{O}$ and $I_{B}$ are values of average (or median) intensity of the object and its close surroundings.

We are calculating the SNR value, as well; in our case this value is lower than unity, because the object is darker than the background. In addition, the graphs of the intensity profiles over the borders of the object can be helpful. The steeper and higher the line is, the more contrast the object has. We used this approach to evaluate the contrast of mitochondria in cells of Euglena gracilis prepared under different procedures using various staining agents (see Fig. 1).

The analysis of the figure is semiautomatic as mentioned above. The manual selection of the ROI (Region of Interest) is required. Selected ROI is eroded by the disc structural element of size 2. This is done because even the manual selection of the borders can be inaccurate. Erosion shrinks little bit the selection but it is more probable that the whole eroded area will be inside the object. This eroded area serves as binary mask of the object. Similar step is done for analysis of the surroundings. The original ROI is dilated by the disc structural element of size 2 . In this case the area is enlarged. This step gives us some tolerance $( \pm 2$ px from original selection) of inaccurate selection of the object's borders. This final mask is dilated by a disc of size 25 . When we subtract these two masks we obtain the surroundings (close neighbourhood) of the object.
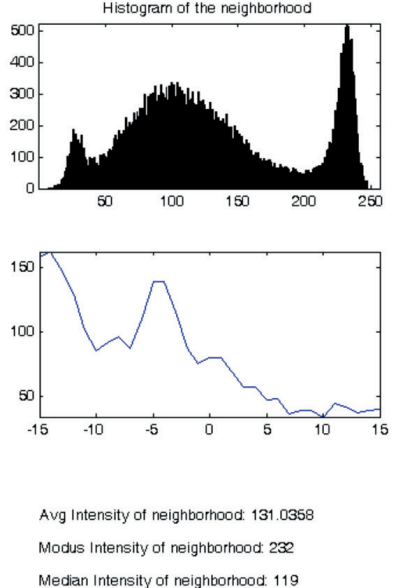
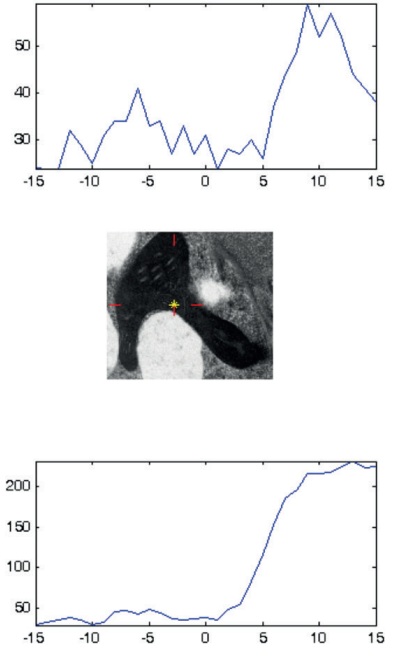
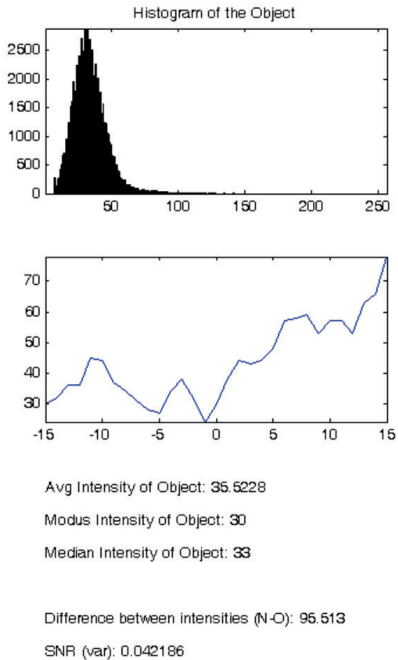

SNR (var): 0.042186

Fig. 2 Analysis of the sample A. Upper row: Histogram of neighborhood, top intensity profile, histogram of the object; Middle row: Left intensity profile, image of ROI with marked center (yellow star) and intensity profiles (red lines), right intensity profile; Bottom row: Measured characteristics of the neighborhood, bottom intensity profile, measured characteristics of the object 

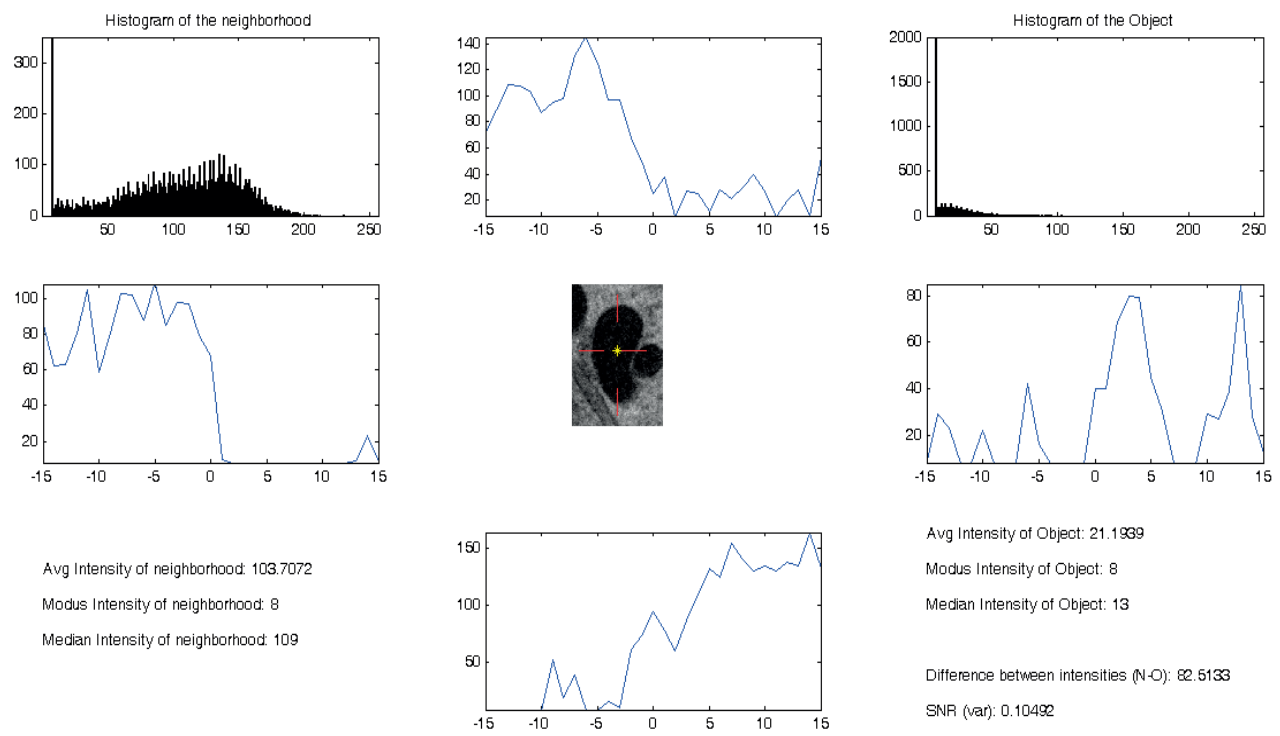

Fig. 3 Analysis of the sample B. Upper row: Histogram of the neighbourhood, top intensity profile, histogram of the object; Middle row: Left intensity profile, image of ROI with marked centre (yellow star) and intensity profiles (red lines), right intensity profile; Bottom row: Measured characteristics of the neighbourhood, bottom intensity profile, measured characteristics of the object

We are plotting the histograms of the neighbourhood and the object to have better impression how the intensities in these areas are represented. There are 4 graphs of the intensity profiles at the borders of the object, as well. We found the centre as a centroid of the object and the profiles are taken horizontally and vertically according to the centre. On these graphs we can see how precisely the object was selected.

\section{Results and discussion}

Measurement of the sample A (same as in Fig. 1A contrasting method $2 \% \mathrm{OsO}_{4}$ and $100 \%$ acetone - is presented in Fig. 2, of the sample B - contrasted with OTO method ( $2 \% \mathrm{OsO}_{4}$, $100 \%$ acetone, thiocarbohydrazide) - in Fig. 3, of the sample C with OTO plus $0.1 \% \mathrm{UA}, \mathrm{Pb}\left(\mathrm{NO}_{3}\right)_{2}$ and methanol - in Fig. 4, and the last sample D - contrasted with Ferrikyanide plus OTO and $0.1 \%$ UA - Fig. 5 .
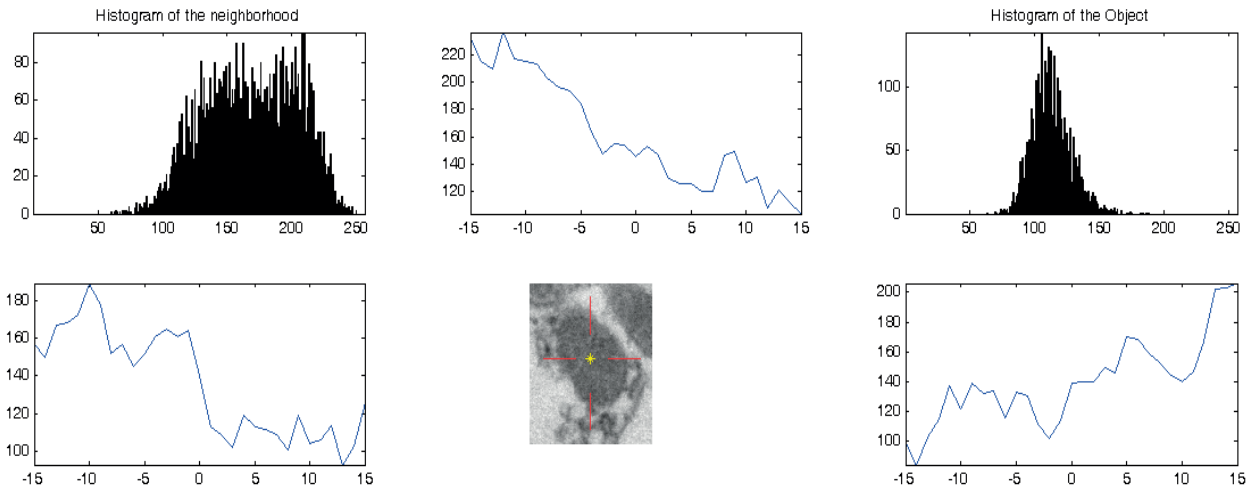

Avg Intensity of neighborhood: 167.8668 Modus Intensity of neighborhood: 208 Median Intensity of neighborhood: 169
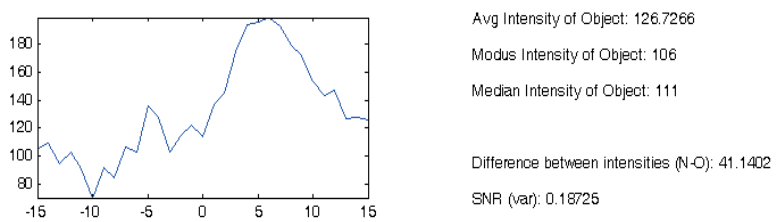

Fig. 4 Analysis of the sample C. Upper row: Histogram of the neighbourhood, top intensity profile, histogram of the object; Middle row: Left intensity profile, image of ROI with marked centre (yellow star) and intensity profiles (red lines), right intensity profile; Bottom row: Measured characteristics of the neighbourhood, bottom intensity profile, measured characteristics of the object 


\section{COMMNICOIIONS}
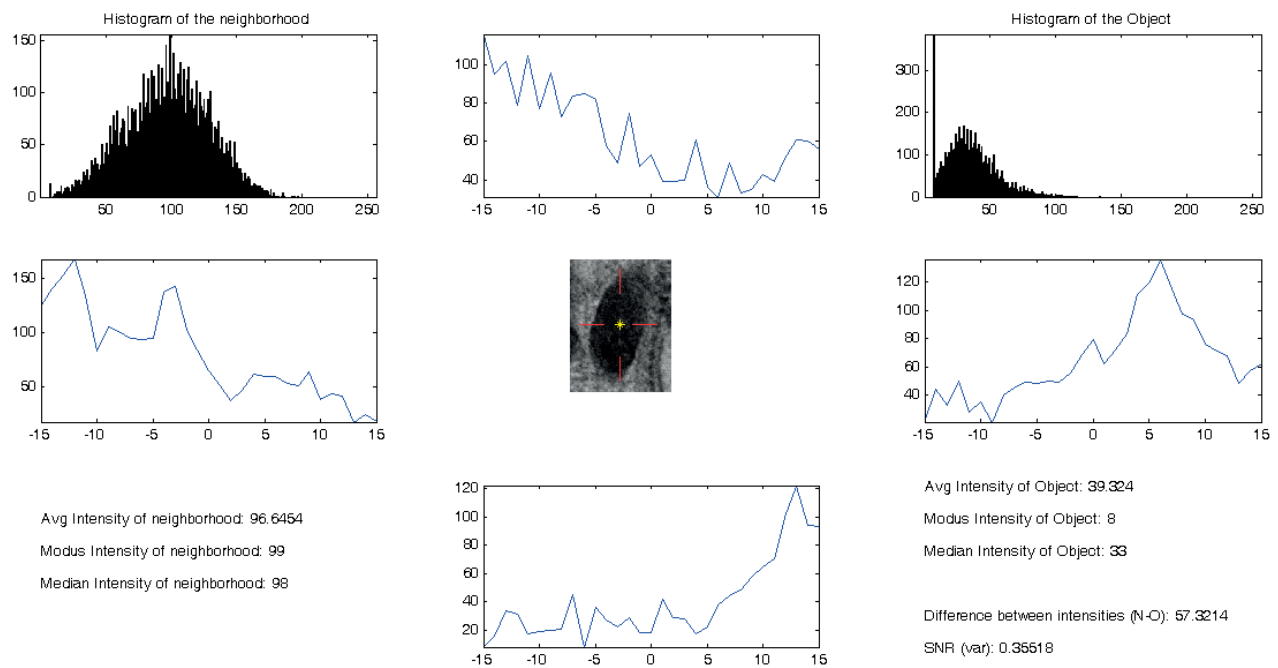

Fig. 5 Analysis of the sample D. Upper row: Histogram of the neighbourhood, top intensity profile, histogram of the object; Middle row: Left intensity profile, image of ROI with marked centre (yellow star) and intensity profiles (red lines), right intensity profile; Bottom row: Measured characteristics of the neighbourhood, bottom intensity profile, measured characteristics of the object

If we looked at images, we can say something about the contrast. The methods (or images) can be sorted by the decreasing contrast by the user probably as B, A, D, C. Maybe someone can swap $\mathrm{B}$ and $\mathrm{A}$ to $\mathrm{A}, \mathrm{B}, \mathrm{D}$ and $\mathrm{C}$. This evaluation is very individual and subjective. Can we find some objectively defined metric, which can be used universaly?

In Table 1 are shown the measured values and in Table 2 are shown the calculated properties and statistical analysis of measured values.

If we looked at values in these tables, we can estimate the order with the decreasing contrast. The first clue can be the differences in the average intensities. This gives us the sequence A, B, D and C. This corresponds to the visual comparison, but we cannot say that the contrast of the sample B is twice higher than in image $\mathrm{C}$, because the numbers represent only the average intensities.

The standard measure is the signal-to-noise ratio. SNR for images is defined as the ratio of the average intensity of the object and standard deviation of the intensity the background. We can also take the value of the signal divided by the value of the noise (background).If we have a look at numbers in Table 2, corresponding to the SNR, we can claim that in this case the SNR is not useful (maybe the SNR calculated with average values in reverse order).

The next measure is the Michelson contrast. The contrast calculated using average or median values gives us sequence B, $\mathrm{A}, \mathrm{D}$ and $\mathrm{C}$. This value is lower than zero, because in formula is intensity of the object minus intensity of the background and the

Measured characteristics of the object and its neighbourhood

Table 1

\begin{tabular}{|c|c|c|c|c|}
\hline Neighborhood and Object characteristics & $\mathbf{A}$ & B & $\mathrm{C}$ & D \\
\hline Average intensity of the neighbourhood & 131.0358 & 103.7072 & 167.8668 & 96.6454 \\
\hline Modus intensity of the neighbourhood & 232 & 8 & 208 & 99 \\
\hline Median intensity of the neighbourhood & 119 & 109 & 169 & 98 \\
\hline STD of the neighbourhood & 62.77 & 45.55 & 35.69 & 31.84 \\
\hline IQR of the neighbourhood & 98 & 66 & 57 & 45 \\
\hline Average intesity of the object & 35.5228 & 21.1939 & 126.7266 & 39.324 \\
\hline Modus intensity of the object & 30 & 8 & 106 & 8 \\
\hline Median intensity of the object & 33 & 13 & 111 & 33 \\
\hline STD of the object & 14.36 & 19.75 & 15.06 & 18.39 \\
\hline IQR of the object & 15 & 20 & 19 & 24 \\
\hline
\end{tabular}


Statistical evaluation of measured characteristics

Table 2

\begin{tabular}{lcccc}
\hline Neighbourhood and Object evaluation & A & B & C & D \\
\hline Difference between avg. intensities (N-O) & 95.513 & 82.5133 & 41.1402 & 57.3214 \\
SNR - standard definition & 0.56592 & 0.465289 & 3.550759 & 1.23505 \\
SNR - variance & 0.042186 & 0.10492 & 0.18725 & 0.35518 \\
SNR - avg. & 0.2710923 & 0.2043629 & 0.7549235 & 0.4068895 \\
SNR - median & 0.2773109 & 0.1192661 & 0.6568047 & 0.3367347 \\
SNR - modus & 0.1293103 & 1 & 0.5096154 & 0.0808081 \\
Contrast - average & -0.57599 & -0.60963 & -0.14257 & -0.41625 \\
Contrast - median & -0.565789 & -0.786885 & -0.207143 & -0.496183 \\
Contrast - modus & -0.770992 & 0 & -0.324841 & -0.850467 \\
\hline
\end{tabular}

background is brighter than the object itself. If the object would be brighter than the background the number would be positive.

Of course, the intensity of the sample can be affected by many variables. We expected in our test that images were taken under the same or almost the same conditions and with the focus on the best images. This means that we try to avoid changes of other conditions except the contrasting technique and changes of the sample.

The shape of histogram of the intensities distribution in the object, as well as STD and IQR values, gives us the knowledge about contrast inside the object, whether we can determine the inner structure or not.

Also comparison of STD (IQR) values of the object and STD (IQR) values of the surroundings can tell us something about the intensity distribution of these parts of the image.

Evaluation of contrast, based on measurement, gives us the opportunity to redesign the experiments to get better image and use automatic image processing of obtained results.

Problem with biological samples (experiments) is that they are not fully repeatable. You need to use different part of the cell, different cut through the sample or completely different cells. Some differences in samples and measurements should be considered as differences in the samples and not in contrasting methods because the organelles do not have the same surroundings in every cell.

\section{Conclusion}

Our analysis brings another view on the local contrast measurement. This analysis can be used, due to manual selection of borders, in cases where the standard algorithms for automatic edge detection fails. As can be seen, objects in the images from the electron microscope can have very fuzzy borders.

Testing of this algorithm was done by using 8 bit images, but it can be used with very small changes for analysis of 12 bit or 16 bit images. The 8 bit images were used because we already had many different images with different contrasting methods which were used for the visual comparison of the contrasting methods.

From comparison of different metrics and different statistics with the visual evaluation of the images, we can claim that for the TEM images and images with similar structure, the changed Michelson contrast (Equation 2) using average or median values, is the appropriate method for local contrast measurement.

\section{Acknowledgement}

I would like to thank to Jana Nebesarova and Marie Vancova for the collaboration and for images. The images were taken on the TEM at the Biology Centre of the Czech Academy of Sciences.

\section{References}

[1] ELLIS, E. A.: Staining Sectioned Biological Specimens for Transmission Electron Microscopy: Conventional and En Bloc Stains. Kuo, J. (Ed.), Electron Microscopy, p. 57-72, Springer, New York, 2014.

[2] ALLISON, D., VON SCHULTHESS, G. K.: The Encyclopedias of Medical Imaging, $2^{\text {nd }}$ ed. Isis Medical Media, New York, 1998.

[3] PELI, E.: Contrast in Complex Images. Journal of the Optical Society of America, 7(10), 2032-2040, 1990.

[4] GONZALEZ, R.S., WINTZ P.: Digital Image Processing. Addison-Wesley Publishing Co., USA, 1977.

[5] MICHELSON, A. A.: Studies in Optics. University of Chicago Press, Chicago, 1927. 